\title{
EMBEDDINGS OF BRAID GROUPS INTO MAPPING CLASS GROUPS AND THEIR HOMOLOGY
}

\author{
Carl-Friedrich Bödigheimer and Ulrike Tillmann
}

\begin{abstract}
We construct several families of embeddings of braid groups into mapping class groups of orientable and non-orientable surfaces and prove that they induce the trivial map in stable homology in the orientable case, but not so in the non-orientable case. We show that these embeddings are nongeometric in the sense that the standard generators of the braid group are not mapped to Dehn twists.
\end{abstract}

\section{Introduction}

Let $\Gamma_{g, n}$ denote the mapping class group of an oriented surface $\Sigma_{g, n}$ of genus $g$ with $n$ parametrized boundary components, i.e., $\Gamma_{g, n}$ is the group of connected components of the group of orientation preserving diffeomorphisms of $\Sigma_{g, n}$ that fix the boundary point-wise. For a simple closed curve $a$ on the surface, let $D_{a}$ denote the Dehn twist around $a$. When two simple closed curves $a$ and $b$ intersect in one point the associated Dehn twists satisfy the braid relation $D_{a} D_{b} D_{a}=D_{b} D_{a} D_{b}$, and if they do not intersect, the corresponding Dehn twists commute $D_{a} D_{b}=D_{b} D_{a}$.

Thus a chain of $n$ interlocking simple closed curves $a_{1}, \ldots, a_{n}$ on some surface defines a map from the braid group $B_{n+1}$ on $n+1$ strands into the mapping class group of a subsurface $\Sigma$ containing the union of theses curves; these mapping classes fix the boundary of $\Sigma$ point-wise. The smallest such subsurface $\Sigma$ is a neighbourhood of the union of the curves. When $n=2 g+1$ is odd, this is $\Sigma_{g, 2}$, and when $n=2 g$ is even, this is $\Sigma_{g, 1}$. Thus we have homomorphisms of groups

$$
\phi: B_{2 g+2} \longrightarrow \Gamma_{g, 2} \quad \text { and } \phi: B_{2 g+1} \longrightarrow \Gamma_{g, 1} .
$$

These are injections by a theorem of Birman and Hilden [BH1], [BH2]. 


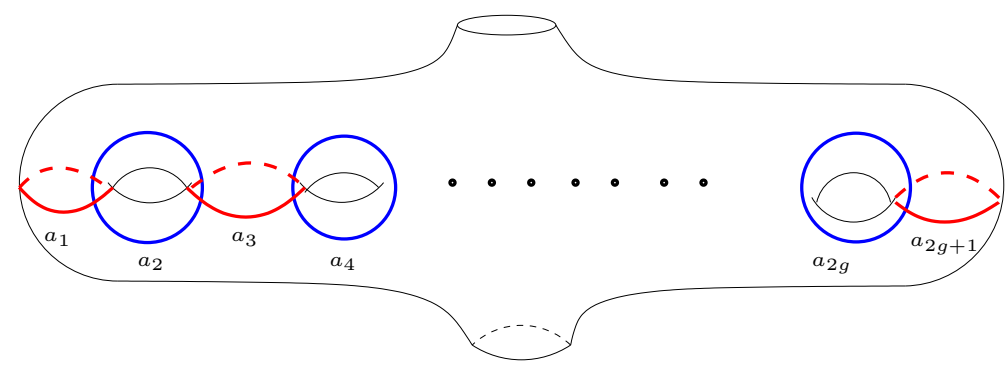

Figure 1: The standard geometric embedding $\phi: B_{2 g+2} \rightarrow \Gamma_{g, 2}$.

Wajnryb [W1] calls such embeddings that send the standard generators of the braid group to Dehn twists geometric. He asks in [W2] whether there are nongeometric embeddings. The first example of such a non-geometric embedding was given by Szepietowski [S]. Our first goal in this paper is to produce many more such non-geometric embeddings and show that they are ubiquitous. We construct these in section 2 and prove in section 3 that they are non-geometric.

Motivated by a conjecture of Harer and following some ideas of F.R. Cohen $[\mathrm{C}]$, in $[\mathrm{SoT}]$ and $[\mathrm{SeT}]$ it was shown that the geometric embedding $\phi$ induces the trivial map in stable homology, that is the map in homology is zero in positive degrees as long as the genus of the underlying surface is large enough relative to the degree. Our second goal here is to show that this is also the case for the non-geometric embeddings constructed in section 2. (For one of these maps, this answers a question left open in [SoT].) To this purpose we show in section 4 how our embeddings from section 2 induce maps of algebras over an $E_{2}$-operad, and deduce in section 5 that all of them induce the trivial map in stable homology. Furthermore, while it may be expected that all maps from a braid group to the mapping class group of an orientable surface will induce the trivial map on stable homology, we show that this is not true for embeddings of a braid group into the mapping class group of a non-orientable surface by explicitly computing the image of one such embedding in stable homology. Finally, in section 6 , we analyse the induced maps in unstable homology. Only partial results are obtained here. In particular, it remains an open question whether $\phi$ induces the trivial map in unstable homology for field coefficients.

Acknowledgement. We would like to thank Blazej Szepietowski for sending his paper and Mustafa Korkmaz for e-mail correspondence.

\section{Non-geometric embeddings}

We will construct various injections of braid groups into mapping class groups. All embeddings that we know of, geometric or not, initially start with the standard identification of the braid groups with mapping class groups. The pure 
braid group on $g$ strands is the mapping class group $\Gamma_{0,1}^{g}$ of a disk with $g$ marked points, and the pure ribbon braid group is the mapping class group $\Gamma_{0, g+1} \simeq \mathbb{Z}^{g} \times \Gamma_{0,1}^{g}$ of a disk with $g$ holes the boundary of which are parametrised. The factors of $\mathbb{Z}$ correspond to the Dehn twists around the boundary circles of the holes. Similarly, the braid group $B_{g}$ can be identified with the mapping class group $\Gamma_{0,1}^{(g)}$ of a disk with $g$ punctures (or $g$ unordered marked points), and the ribbon braid group $\mathbb{Z} \imath B_{g}$ with the mapping class group $\Gamma_{0,(g), 1}$ of the disk with $g$ unordered holes for which the underlying diffeomorphisms may in a parametrisation preserving way interchange the boundaries of the holes and fix the outer boundary curve point-wise. Note that the resulting inclusion

$$
\gamma: B_{g} \hookrightarrow \mathbb{Z} \curlywedge B_{g} \simeq \Gamma_{0,(g), 1}
$$

maps the standard generator that interchanges the $i$-th and $(i+1)$-st strand in the braid group to half of the Dehn twists around a simple closed curve enclosing the $i$-th and $(i+1)$-st holes followed by a half Dehn twist around each of these holes in the opposite direction; see Figure 2.

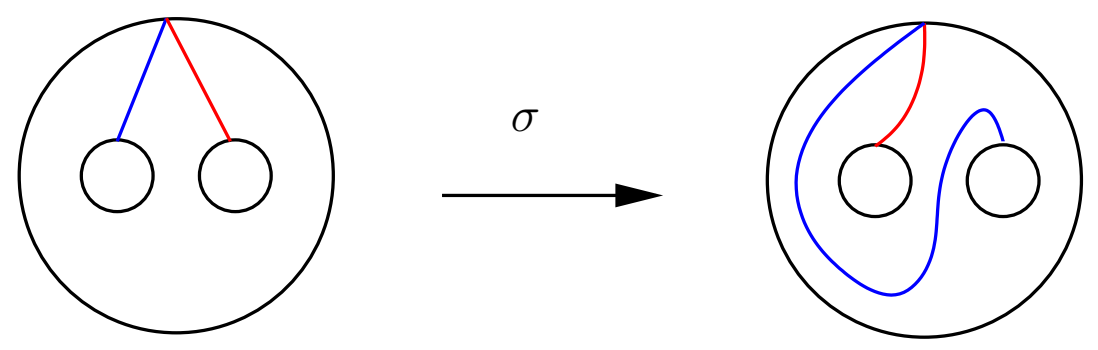

Figure 2: Image of a generator $\sigma \in B_{g}$ under $\gamma$.

Clearly, any genus zero subsurface $\Sigma_{0, g+1}$ of a surface $\Sigma$ defines for us a map from the pure ribbon braid group $\Gamma_{0, g+1}$ into the mapping class group of $\Sigma$. Not all such maps, however, can be extended to the ribbon braid group $\Gamma_{0,(g), 1}$. Below we explore various constructions of surfaces from $\Sigma_{0, g+1}$ that allow such an extension.

\subsection{Mirror construction.}

Our first example of a non-geometric embedding was also considered in [SoT].

We double the disk with $g$ holes by reflecting it in a plane containing the boundary circles of the holes to obtain an oriented surface $\Sigma_{g-1,2}$ as indicated in Figure 3, and extend diffeomorphisms of the disk with holes to $\Sigma_{g-1,2}$ by reflection in the plane. This defines a map on mapping class groups

$$
R: \Gamma_{0,(g), 1} \longrightarrow \Gamma_{g-1,2} .
$$


Precomposing $R$ with the natural inclusion $B_{g} \subset \Gamma_{0,(g), 1}$ defines the map

$$
B_{g} \stackrel{\gamma}{\longrightarrow} \Gamma_{0,(g), 1} \stackrel{R}{\longrightarrow} \Gamma_{g-1,2} .
$$

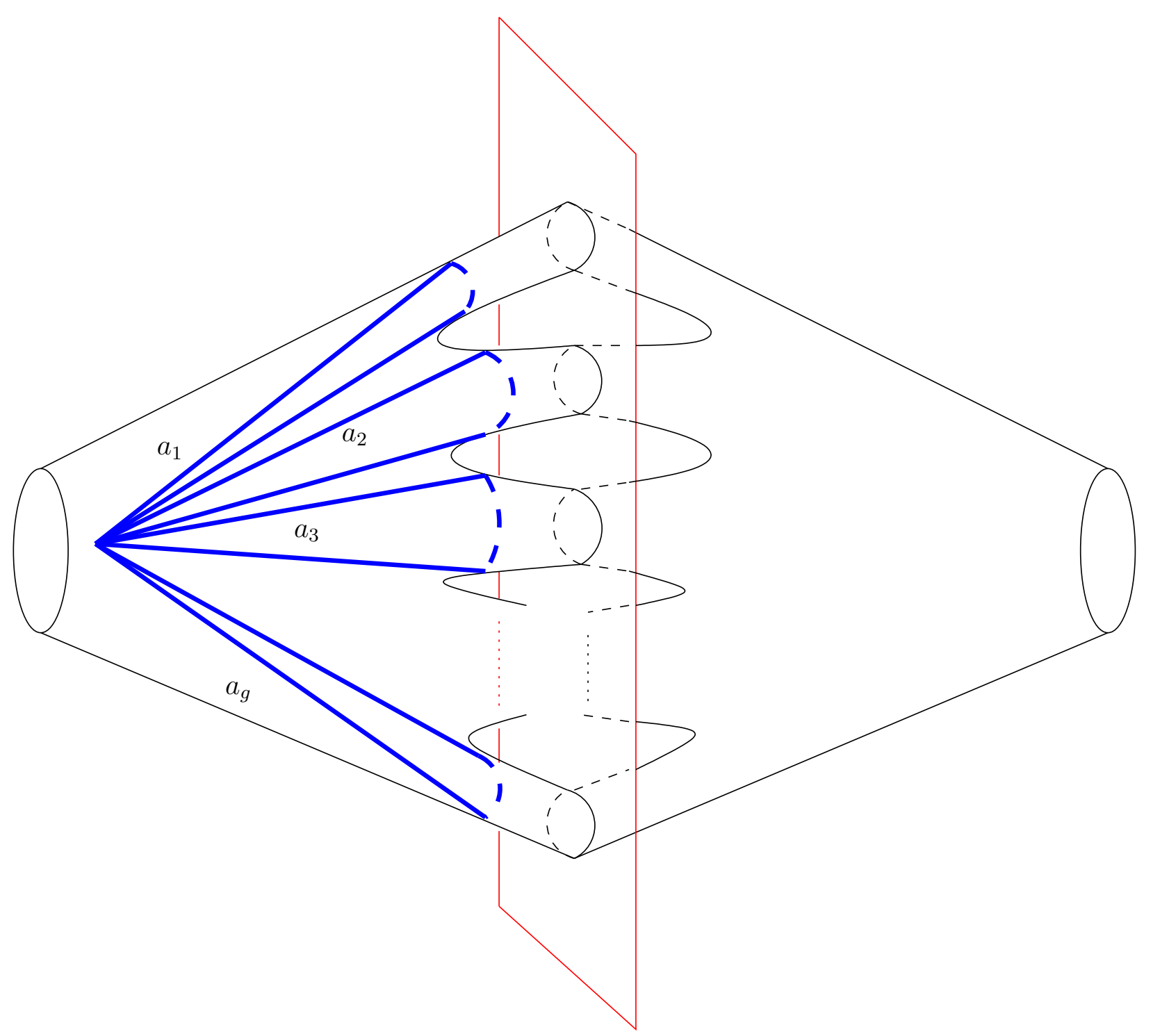

Figure 3: Mirror construction $R$ and subgroup $\left\langle a_{1}, \ldots, a_{g}\right\rangle \subset \pi_{1}\left(\Sigma_{g-1,2}\right)$.

Lemma 2.1. The composition $R \circ \gamma$ is an injection.

Proof. We are going to detect the elements in the image by their action on the fundamental group of the underlying surface. Recall, the action of the 
braid group on the fundamental group of the disk with $g$ holes defines Artin's inclusion [A] of the braid group into the automorphism group of $\pi_{1}\left(\Sigma_{0, g+1}\right)=$ $F_{g}=\left\langle a_{1}, \ldots, a_{g}\right\rangle$, a free group of rank $g$ :

$$
A: B_{g} \hookrightarrow \operatorname{Aut}\left(F_{g}\right) .
$$

The fundamental group of $\Sigma_{0, g+1}$ in turn injects into the fundamental group of $\Sigma_{g-1,2}$. Indeed, each standard generator of $F_{g}=\left\langle a_{1}, \ldots, a_{g}\right\rangle$ is mapped to the corresponding generator of $\pi_{1}\left(\Sigma_{g-1,2}\right)$ which is the free group $F_{2 g-1}=$ $\left\langle a_{1}, b_{1}, \ldots, b_{g-1}, a_{g}\right\rangle$ of rank $2 g-1$; see Figure 3 .

The action of diffeomorphisms on the fundamental group of the surface induces a group homomorphisms $\Gamma_{g-1,2} \rightarrow \operatorname{Aut}\left(F_{2 g-1}\right)$, and by restricting to the subgroup $F_{g}=\left\langle a_{1}, \ldots, a_{g}\right\rangle$ a map to the set $\operatorname{Hom}\left(F_{g}, F_{2 g-1}\right)$. We follow this map by the projection map to the monoid $\operatorname{Hom}\left(F_{g}, F_{g}\right)$, induced from the group homomorphisms $F_{2 g-1} \rightarrow F_{g}$ that maps each $a_{i}$ to itself and each $b_{i}$ to the identity element. We have the following commutative diagram; note that the right vertical arrow is only a map of sets.

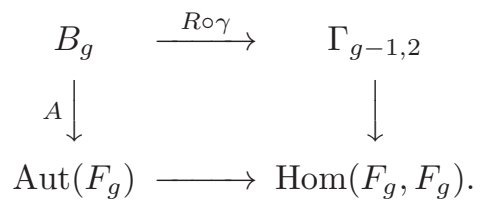

As $A$ and the bottom horizontal map are injective this shows that $R \circ \gamma$ is injective.

\subsection{Szepietowski's construction.}

We next recall the construction from [S]. Starting with $\Sigma_{0, g+1}$, the disk with $g$ holes, we glue to the boundary of each hole a Möbius band $N_{1,1}$ so that the resulting surface is a non-orientable surface $N_{g, 1}$ of genus $g$ with one parametrised boundary component. Any diffeomorphisms $f: \Sigma_{0, g+1} \rightarrow \Sigma_{0, g+1}$ which fixes the boundary pointwise can be extended across the boundary by the identity of $N_{1,1} \sqcup \ldots \sqcup N_{1,1}$ and gives thus a diffeomorphism of $N_{g, 1}$. This defines (via $\gamma$ ) a homomorphism from the pure braid group to the mapping class group of $N_{g, 1}$. This latter homomorphism can be extended to a map from the whole braid group as follows: extend a diffeomorphism $f$ which permutes the $g$ inner boundaries by the corresponding permutation diffeomorphism of $N_{1,1} \sqcup \ldots \sqcup N_{1,1}$. This defines

$$
\varphi: B_{g} \longrightarrow \mathcal{N}_{g, 1},
$$

where $\mathcal{N}_{g, 1}$ denotes the mapping class group of $N_{g, 1}$. By a result of Birman and Chillingworth [BC1], [BC2], extended to surfaces with boundary in [S], the lift 
of diffeomorphisms of a non-orientable surface to its double cover induces an injection of mapping class groups:

$$
L: \mathcal{N}_{g, 1} \hookrightarrow \Gamma_{g-1,2}
$$

Lemma 2.2. The composition $L \circ \varphi$ is an injection.

Proof. We give an alternative proof to the one in [S]. As in the proof of Lemma 2.1 we consider the action of the mapping class group on the fundamental group of the underlying surfaces. In this case the fundamental group of the disk with $g$ holes $\pi_{1}\left(\Sigma_{0, g+1}\right)=F_{g}=\left\langle a_{1}, \ldots, a_{g}\right\rangle$ injects into the fundamental group $\pi_{1}\left(N_{g, 1}\right)=F_{g}=\left\langle c_{1}, \ldots, c_{g}\right\rangle$ by sending $a_{i}$ to $2 c_{i}$. As by [A] the action of the braid group on the subgroup $\left\langle a_{1}, \ldots, a_{g}\right\rangle$ is faithful it will be so on the whole group. Hence $\varphi$ is injective, and so is $L \circ \varphi$.

\subsection{Geometric embedding and orientation cover.}

The closed non-orientable surface $N_{2 g+1}$ can be obtained by sewing a Möbius band to the orientable surface $\Sigma_{g, 1}$.

Lemma 2.3. The inclusion of surfaces $\Sigma_{g, 1} \subset N_{2 g+1}$ induces an inclusion of mapping class groups

$$
\Gamma_{g, 1} /\left\langle D_{\partial}\right\rangle \simeq \Gamma_{g}^{1} \hookrightarrow \mathcal{N}_{2 g+1} .
$$

Here $D_{\partial}$ denotes the Dehn twist around the boundary curve in $\Sigma_{g, 1}$. This is a central element in $\Gamma_{g, 1}$. We identify the quotient group with the mapping class group of a surface with a marked point (or a surface with one parametrized boundary component).

Remark. This subgroup of $\mathcal{N}_{2 g+1}$ can be identified with the point-wise stabiliser of the core of the Möbius band that has been sewn on to $\Sigma_{g, 1}$ to form $N_{2 g+1}$. For $g>1$ this subgroup has infinite index but has the same virtual cohomological dimension as the whole group [I],

$$
\operatorname{vcd}\left(\Gamma_{g}^{1}\right)=4 g-3=\operatorname{vcd}\left(\mathcal{N}_{2 g+1}\right) .
$$

Proof. It is an elementary fact that the Dehn twist around the boundary of a Möbius strip is isotopic to the identity. This implies that $D_{\partial}$ is in the kernel of $\Gamma_{g, 1} \rightarrow \mathcal{N}_{2 g+1}$. To show that $D_{\partial}$ generates the kernel, consider the composition

$$
\Gamma_{g, 1} \longrightarrow \mathcal{N}_{2 g+1} \stackrel{L}{\hookrightarrow} \Gamma_{2 g} .
$$

Let $J$ denote the fix-point free orientation reversing involution of $\Sigma_{2 g}$ with quotient $N_{2 g+1}$. As in [BC], we embed $\Sigma_{2 g}$ in $\mathbb{R}^{3}$ symmetrically around the 
origin and take $J=-$ Id to be the reflection through the origin, see Figure 4. The image of any element $x \in \Gamma_{g, 1}$ is the product of $\bar{x}$ with $J \bar{x} J$, where $\bar{x}$ acts on the left side of the surface via $x$ and via the identity on the right side. So if $x$ is in the kernel then $\bar{x}^{-1}=J \bar{x} J$ in $\Gamma_{2 g}$. But $\bar{x}^{-1}$ can be represented by a diffeomorphism with support entirely in the left half of the surface and $J \bar{x} J$ by a diffeomorphism with support entirely in the right half of the surface. As the diffeomorphisms are isotopic, so must be their supports. Hence, $\bar{x}$ has a representing diffeomorphism supported in a tubular neighbourhood of the boundary, i.e. $x \in\left\langle D_{\partial}\right\rangle$.

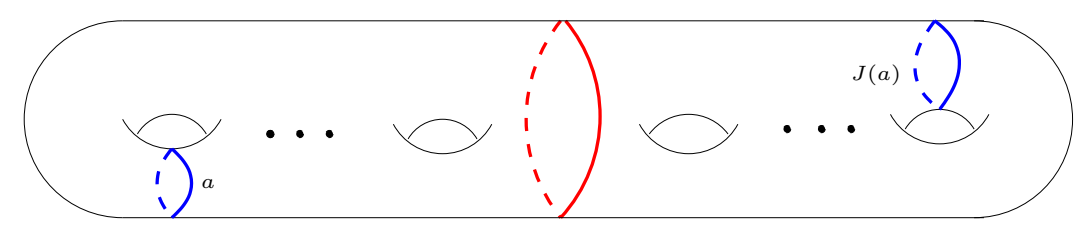

Figure 4: The involution $J=-\operatorname{Id}$ in $\mathbb{R}^{3}$.

Our third construction is the composition

$$
L^{\prime} \circ \phi: B_{2 g} \stackrel{\phi}{\hookrightarrow} \Gamma_{g-1,2} \hookrightarrow \Gamma_{g}^{1} \longrightarrow \mathcal{N}_{2 g+1} \stackrel{L}{\longrightarrow} \Gamma_{2 g} .
$$

The unlabelled map $\Gamma_{g-1,2} \rightarrow \Gamma_{g}^{1}$, which is induced by gluing a pair of pants to the two boundary circles of $\Sigma_{g-1,2}$, is an inclusion, see [PR]. Thus, again we have constructed an embedding.

\subsection{Geometric embedding and mirror construction.}

Similarly to our third example, we may combine the geometric embedding $\phi$ : $B_{2 g} \rightarrow \Gamma_{g-1,2}$ with a mirror construction. For this first glue a torus $\Sigma_{1,2}$ along one of its boundary circles to $\Sigma_{g-1,2}$ and embed the other boundary circle in the plane. Now double the resulting surface $\Sigma_{g, 2}$ by reflection in the plane to yield a surface $\Sigma_{2 g, 2}$. We leave it as an exercise to prove the following result.

Lemma 2.4. The composition $B_{2 g} \stackrel{\phi}{\longrightarrow} \Gamma_{g-1,2} \longrightarrow \Gamma_{g, 2} \stackrel{R^{\prime}}{\longrightarrow} \Gamma_{2 g, 2}$ is an injection.

\subsection{Operadic embedding.}

The following embedding is well-known as part of an $E_{2}$-operad action, compare section 4 .

Starting with a disk $\Sigma_{0, g+1}$ with $g$ holes we glue a torus $\Sigma_{1,1}$ with one disk removed to each of boundaries of the $g$ holes of the disk. The result is a surface 
$\Sigma_{g, 1}$. As in the construction of $\varphi$ in (2.3) we may extend diffeomorphisms of the disk via the identity to the glued on tori to define a map

$$
\varphi^{+}: B_{g} \longrightarrow \Gamma_{g, 1} \text {. }
$$

Lemma 2.5. The map $\varphi^{+}$is an injection.

Proof. The fundamental group of $\Sigma_{0, g+1}$ is freely generated by the $g$ curves $c_{1}, \ldots, c_{g}$ that start at a point on the outside boundary and wind around one of the holes: $\pi_{1}\left(\Sigma_{0, g+1}\right)=\left\langle c_{1}, \ldots, c_{g}\right\rangle$. The fundamental group of $\Sigma_{g, 1}$ is freely generated by $2 g$ curves: $\pi_{1}\left(\Sigma_{g, 1}\right)=\left\langle a_{1}, b_{1}, \ldots, a_{g}, b_{g}\right\rangle$ with $a_{i}$ and $b_{i}$ the standard generators in the fundamental group of the $i$-th copy of the torus. Then under the inclusion of the disk into the genus $g$ surface the generator $c_{i}$ maps to $a_{i} b_{i} a_{i}^{-1} b_{i}^{-1}$. So the $c_{i}$ are mapped to words on subsets of the alphabet that are disjoint. Hence their images generate a free group on $g$ generators and the induced map on fundamental groups is therefore an injection. We argue as before, that therefore the action of $B_{g}$ via $\varphi^{+}$on $\pi_{1}\left(\Sigma_{g, 1}\right)$ remains faithful and hence $\varphi^{+}$must be an injection.

\subsection{More constructions.}

Other inclusions of the braid group into mapping class groups can be constructed from the above ones by precomposing with an automorphism of the braid group or composition with an automorphism of the mapping class groups. We note here that conjugation of a non-geometric (or geometric) embedding by a mapping class yields again a non-geometric (or geometric) embedding as Dehn twists are conjugated to Dehn twists. Thus these will indeed produce new examples of non-geometric embeddings (or geometric ones). We also note that conjugation by a fixed element induces the identity in homology. The results of section 5 and section 6 are therefore also valid for these variations. We will not mention these additional embeddings any further.

\section{Proving non-geometricity}

All of our constructions in section 2, with the exception of $\varphi^{+}$in (2.5), use an orientation reversing diffeomorphism of the oriented surface associated to the target. This is key for proving that these embeddings are not geometric.

Lemma 3.1. Let $J$ be an orientation reversing involution of $\Sigma_{g, n}$ and $x \in \Gamma_{g, n}$ commute with $J$. Then $x$ is not a power of a Dehn twist unless it is trivial. 
Proof. We borrow an argument from [S]. Assume $x$ is the $k$-th power of a Dehn twist $D_{c}$ around a simple closed curve $c$ in $\Sigma_{g, n}$. As $x$ commutes with $J$ and $J$ is orientation reversing,

$$
x=D_{c}^{k}=J D_{c}^{k} J=D_{J(c)}^{-k} .
$$

But this identity can only hold if $c$ is isotopic to $J(c)$ and $k=-k$. Therefore $x=D_{c}^{k}$ is trivial.

Theorem 3.2. The embeddings $R \circ \gamma, L \circ \varphi, L^{\prime} \circ \phi$ and $R^{\prime} \circ \phi$ are not geometric.

An embedding that sends the standard generators of the braid group to some powers of Dehn twists are also called pseudo-geometric [W2]. The proof of the theorem will show that these maps are not even pseudo-geometric.

Proof. Consider $R \circ \gamma$ and let $\sigma$ be the image under $\gamma$ of one of the standard generators of the braid group. The image $R(\sigma)$ is by definition invariant under the reflection in the plane (see Figure 3 ) which is orientation reversing. Lemma 3.1 implies that $R(\sigma)$ cannot be a power of a Dehn twist. The arguments for $L \circ \varphi, L^{\prime} \circ \phi$ and $R^{\prime} \circ \phi$ are similar.

Remark. The geometric embedding $\phi: B_{2 g+2} \rightarrow \Gamma_{g, 2}$ can also be constructed by a 'doubling' procedure as the maps above. For this, identify first the braid group $B_{2 g+2}$ as the mapping class group of a disk with $2 g+2$ unordered marked points which in turn we identify as the orbit space of a genus $g$ surface $\Sigma_{g, 2}$ under the hyper-elliptic involution, see for example [SeT]. However, in this case the involution is orientation preserving and the construction leads to a geometric inclusion.

We now turn to the standard embedding $\gamma$ from (2.1) and the operadic embedding $\varphi^{+}$constructed in (2.5).

Theorem 3.3. The embeddings $\gamma$ and $\varphi^{+}$are neither geometric nor pseudogeometric.

Proof. Let $\sigma$ be one of the standard generators for the braid group $B_{g}$ and consider its image under $\gamma$. This is a mapping class supported on a disk $\Sigma_{0,3}$ with two holes; compare Figure 2. An application of the Jordan Curve Theorem shows that there are only three non-contractible non-isotopic simple closed curves on $\Sigma_{0,3}$, each isotopic to one of the boundary circles. It is straight forward to check that $\gamma(\sigma)$ is not isotopic to (a power of) a Dehn twist around any of these three curves. Hence, $\gamma$ is not geometric (or pseudo-geometric).

We now turn to $\varphi^{+}$. By definition (2.5), it is the composition of $\gamma$ and the map induced by the inclusion of surfaces $\Sigma_{0, g+1} \subset \Sigma_{g, 1}$ achieved by sewing a torus with one boundary component to each of the interior boundaries of $\Sigma_{0, g+1}$. Thus $\varphi(\sigma)$ is still defined as pictured in Figure 2 and supported by the same $\Sigma_{0,3}$, now a subsurface of $\Sigma_{g, 1}$. 
The support of any Dehn twist $D_{a}$ around a simple closed curve $a$ is a neighbourhood of $a$. Thus, if $\varphi(\sigma)=D_{a}^{k}$ for some $a$ and $k \in \mathbb{N}$, we must be able to isotope the curve $a$ into $\Sigma_{0,3}$. But the argument above still applies and shows that $\varphi^{+}(\sigma)$ cannot be (a power of) a Dehn twist of any curve in $\Sigma_{0,3}$. Hence $\varphi^{+}$is not geometric and not pseudo-geometric.

\section{Action of the braid group operad}

Consider the following group level version of the well-known $E_{2}$-operad. (See, e.g. [T1] for details.)

As in the introduction to section 2, identify the pure braid group on $k$ strands with a subgroup $\mathcal{D}_{k} \subset \Gamma_{0, k+1}$ of the pure ribbon braid group, i.e. the mapping class group of a disk with $k$ holes whose boundaries are parametrised. The collection $\mathcal{D}=\left\{\mathcal{D}_{k}\right\}_{k \geq 0}$ forms an operad with structure maps

$$
\theta: \mathcal{D}_{k} \times\left(\mathcal{D}_{m_{1}} \times \cdots \times \mathcal{D}_{m_{k}}\right) \longrightarrow \mathcal{D}_{m_{1}+\cdots+m_{k}}
$$

induced by sewing of the underlying surfaces. To be more precise, for each $i$, the boundary of the $i$-th hole in $\Sigma_{0, k+1}$ is sewn to the (outer) boundary of the $i$-th disk $\Sigma_{0, m_{i}+1}$.

The operad $\mathcal{D}$ acts naturally on $\mathcal{B}=\coprod_{m \geq 1} B_{m}$ where each braid group $B_{m}$ is identified via $\gamma$ as a subgroup of $\Gamma_{0,(m), 1}$. The action is again induced by gluing of the underlying surfaces. Indeed

$$
\theta_{\mathcal{B}}: \mathcal{D}_{k} \times\left(B_{m_{1}} \times \cdots \times B_{m_{k}}\right) \longrightarrow B_{m_{1}+\cdots+m_{k}}
$$

agrees with the structure map $\theta$ on the pure braid subgroups.

This action of $\mathcal{D}$ can further be extended to an action on

$$
\Gamma_{R}=\coprod_{m>1} \Gamma_{m-1,2}
$$

via the mirror construction $R$ from (2.2). To define

$$
\theta_{R}: \mathcal{D}_{k} \times\left(\Gamma_{m_{1}-1,2} \times \cdots \times \Gamma_{m_{k}-1,2}\right) \longrightarrow \Gamma_{m_{1}+\cdots+m_{k}-1,2}
$$

place each of the underlying surfaces across a plane, so that one half is reflected by the plane onto the other, as in Figure 3. Then sewing $k$-legged trousers to the $k$ boundary components on the left halves and another one to the right halves gives a surfaces of type $\Sigma_{m_{1}+\cdots+m_{k}-1,2}$. An element in $\mathcal{D}_{k}$ defines a mapping class on the left $k$-legged trousers and by mirroring a class on the right $k$-legged trousers. The following result holds by construction.

Lemma 4.1. The map $R \circ \gamma$ induces a map of $\mathcal{D}$-algebras $\mathcal{B} \rightarrow \Gamma_{R}$. 
Similarly, $\mathcal{N}:=\coprod_{m>1} \mathcal{N}_{m, 1}$ is a $\mathcal{D}$-algebra. Again, the action

$$
\theta_{\mathcal{N}}: \mathcal{D}_{k} \times\left(\mathcal{N}_{m_{1}, 1} \times \cdots \times \mathcal{N}_{m_{k}, 1}\right) \longrightarrow \mathcal{N}_{m_{1}+\cdots+m_{k}, 1}
$$

is induced by sewing the legs of $k$-legged trousers to the boundary components of the $k$ non-orientable surfaces. And again by construction, we obtain the following result.

Lemma 4.2. The map $\varphi$ induces a map of $\mathcal{D}$-algebras $\mathcal{B} \rightarrow \mathcal{N}$.

To see that the lift $L$ to the orientation cover is a map of $\mathcal{D}$-algebras we need to consider a variant $\Gamma_{L}$ of the $\mathcal{D}$-algebra $\Gamma_{R}$. The underlying groups are the same but the action $\theta_{L}$ is such that it commutes with $L$. To achieve this the mapping class defined on one $k$-legged trousers is paired with that on the other via the lift $L$ so that the following holds.

Lemma 4.3. The map $L$ induces a map of $\mathcal{D}$-algebras $\mathcal{N} \rightarrow \Gamma_{L}$.

We recall from [SeT] that also the standard embedding $\phi$ from (1.1) induces a map of $\mathcal{D}$-algebras $\mathcal{B}^{e v} \rightarrow \Gamma_{\phi}$. Here $\mathcal{B}^{e v}=\coprod_{m>1} B_{2 m}$ is a $\mathcal{D}$-subalgebra of $\mathcal{B}$, and $\Gamma_{\phi}$ is the same collection of groups as $\Gamma_{R}$ and $\Gamma_{L}$ but has a slightly different action. We think of $\phi$ as explained in the remark following Theorem 3.2 above as lifting mapping classes of the $2 m$-punctured disk to the ramified double cover $\Sigma_{m-1}$ associated to the hyper-elliptic involution. Thus, in this case $\theta_{\phi}$ is defined so as to commute with the hyper-elliptic involution.

Lemma 4.4. The map $\phi$ induces a map of $\mathcal{D}$-algebras $\mathcal{B}^{e v} \rightarrow \Gamma_{\phi}$.

And we also recall the best-known $\mathcal{D}$-algebra structure on

$$
\Gamma:=\coprod_{m \geq 0} \Gamma_{m, 1} ;
$$

see $[\mathrm{M}]$ and also [Bö]. In this case the action $\theta_{\Gamma}$ is defined just as for $\theta_{\mathcal{N}}$ but with $\mathcal{N}_{m_{i}, 1}$ replaced by $\Gamma_{m_{i}, 1}$. As $\varphi^{+}$is essentially part of the $\mathcal{D}$-algebra structure the following is immediate.

Lemma 4.5. The map $\varphi^{+}$induces a map of $\mathcal{D}$-algebras $\mathcal{B} \rightarrow \Gamma$.

\section{The maps in stable homology}

We will determine the induced maps in stable homology of all the embeddings of braid groups into mapping class groups of orientable or non-orientable surfaces constructed earlier.

The Harer-Ivanov homology stability theorem states that the embedding $\Sigma_{g, 1} \rightarrow \Sigma_{g+1,1}$ induces an isomorphism 


$$
H_{*}\left(\Gamma_{g, 1}\right) \longrightarrow H_{*}\left(\Gamma_{g+1,1}\right)
$$

in a range of degrees, called the stable range. This range has recently been improved to $* \leq 2(g-1) / 3$ by Boldsen [Bo] and Randal-Williams [RW].

Theorem 5.1. In the stable range, the maps $R \circ \gamma, L \circ \varphi, \phi, \varphi^{+}, L^{\prime} \circ \phi$, and $R^{\prime} \circ \phi$ induce the zero map in any reduced, generalised homology theory.

Proof. We sketch the argument here and refer for more details to [SoT], [SeT]. In section 4 we showed that the maps $R \circ \gamma, L \circ \varphi, \phi$ and $\varphi^{+}$are maps of $\mathcal{D}$-algebras. After taking classifying spaces and group completion they induce therefore maps of double loop spaces 1

$$
\Omega B\left(\coprod_{g>0} B\left(B_{g}\right)\right) \simeq \mathbb{Z} \times B\left(B_{\infty}\right)^{+} \simeq \Omega^{2} S^{2} \longrightarrow \Omega B\left(\coprod_{g \geq 0} B \Gamma_{g, 1}\right) \simeq \mathbb{Z} \times B \Gamma_{\infty}^{+} .
$$

Here $B_{\infty}=\lim _{g \rightarrow \infty} B_{g}$ and $\Gamma_{\infty}=\lim _{g \rightarrow \infty} \Gamma_{g, 1}$ are the infinite braid and mapping class groups and $X^{+}$denotes the Quillenization of the space $X$. As $B\left(B_{\infty}\right)^{+} \simeq \Omega_{0}^{2} S^{2} \simeq \Omega^{2} S^{3}$ is the free object on the circle in the category of double loop spaces, on a connected component these maps are determined by their restriction to the circle. But these restrictions have to be homotopic to the constant map as $B\left(\Gamma_{\infty}\right)^{+}$is simply connected. Hence maps $B\left(B_{\infty}\right)^{+} \rightarrow B\left(\Gamma_{\infty}\right)^{+}$ that are maps of double loop spaces are null-homotopic. In particular, they induce the zero map in any reduced, generalised homology theory. Finally, the mapping class groups satisfy (ordinary) homology stability. By an application of the Atiyah-Hirzebruch spectral sequence, the statement of the theorem follows for the first four maps, including $\phi$, and hence for $L^{\prime} \circ \phi$ and $R^{\prime} \circ \phi$.

We now turn our attention to the mapping class group of non-orientable surfaces and the embedding $\varphi: B_{g} \rightarrow \mathcal{N}_{g, 1}$ defined in (2.3). The commutator subgroup of $\mathcal{N}_{g}$ is generated by Dehn twists around two-sided curves. For $g \geq 7$ it has index two and thus $H_{1}\left(\mathcal{N}_{g}\right)=\mathbb{F}_{2}$ and is in particular not trivial; see [K].

The mapping class groups $\mathcal{N}_{g}$ also satisfy homology stability:

$$
H_{*}\left(\mathcal{N}_{g}\right)=H_{*}\left(\mathcal{N}_{g, 1}\right)=H_{*}\left(\mathcal{N}_{g+1,1}\right) .
$$

Here $* \leq(g-3) / 3$ for the first equality and $* \leq g / 3$ for the second, see [Wa2] and $[\mathrm{RW}$.

If $\sigma$ is a standard generator of the braid group, its image under $\varphi$ interchanges two cross caps in $N_{g, 1}$. Therefore, it is not in the index two subgroup of $\mathcal{N}_{g, 1}$ generated by Dehn twists around two-sided curves. Indeed, the product of $\varphi(\sigma)$ with the Dehn twist around the two-sided curve that goes once through each cross cap is a cross-cap slide, see $[\mathrm{S}]$. Hence, the map induced by $\varphi$ on the first homology groups is surjective and not trivial. More generally we have the following result.

\footnotetext{
1 The group completion of $\coprod_{m \geq 1} B\left(B_{2 m}\right)$ consists of all the even components in $\Omega^{2} S^{2}$, and the argument goes through.
} 
Theorem 5.2. Let $g \geq 7$ and $0<* \leq g / 3$. When $\mathbb{F}=\mathbb{Q}$ or $\mathbb{F}=\mathbb{F}_{p}$ for an odd prime $p$, the map

$$
\varphi_{*}: H_{*}\left(B_{g} ; \mathbb{F}\right) \longrightarrow H_{*}\left(\mathcal{N}_{g, 1} ; \mathbb{F}\right)
$$

is zero, while for $\mathbb{F}=\mathbb{F}_{2}$ it is an injection.

Proof. The basic idea of the proof is similar to that used in Theorem 5.1 but we need to use also some quite technical results from [Wa], [T1] and [T2]. We sketch the argument.

It is well-known that the map from the braid to the symmetric group induces after taking classifying spaces, stabilisation and Quillenization the canonical map

$$
\mathbb{Z} \times B B_{\infty}^{+} \simeq \Omega^{2} S^{2} \longrightarrow \Omega^{\infty} S^{\infty} \simeq \mathbb{Z} \times B \Sigma_{\infty}^{+}
$$

from the free object generated by $S^{0}$ in the category of double loop spaces to the corresponding one in the category of infinite loop spaces. In homology with $\mathbb{F}_{2}$ coefficients it induces an inclusion and it is zero in reduced homology with field coefficients of characteristic other than 2 .

By the main theorem of [T1], the double loop space structure on $\mathbb{Z} \times B \mathcal{N}_{\infty}^{+}$ defined by the $\mathcal{D}$-algebra structure on $\mathcal{N}$ extends to an infinite loop space structure. This implies that the map $\Omega^{2} S^{2} \rightarrow \mathbb{Z} \times B \mathcal{N}_{\infty}^{+}$induced by $\varphi$ factors through $\Omega^{\infty} S^{\infty}$ via the above map.

Using cobordism categories of non-orientable surfaces one can show that there is another infinite loop space structure on $\mathbb{Z} \times B \mathcal{N}_{\infty}^{+}$. By a theorem of Wahl these two infinite loop space structures are the same up to homotopy. To be more precise, Wahl shows in [Wa1] that the two constructions lead to the same infinite loop space structures up to homotopy in the orientable case, i.e., for $\mathbb{Z} \times B \Gamma_{\infty}^{+}$. Her argument goes through verbatim to prove the same result for non-orientable surfaces. Thus, the map of infinite loop spaces $\Omega^{\infty} S^{\infty} \rightarrow \mathbb{Z} \times B \mathcal{N}_{\infty}^{+}$here is up to homotopy the same as the one used in [T2]. In [T2] we showed however that this map has a splitting up to homotopy and, in particular, induces an injection in homology.

Combining all this we have proved that the composition

$$
\mathbb{Z} \times B B_{\infty}^{+} \simeq \Omega^{2} S^{2} \longrightarrow \Omega^{\infty} S^{\infty} \longrightarrow \mathbb{Z} \times B \mathcal{N}_{\infty}^{+}
$$

induces an injection on $\mathbb{F}_{2}$-homology and is trivial in reduced homology for $\mathbb{F}=\mathbb{Q}$ or $\mathbb{F}_{p}, p$ odd. As $B_{g} \rightarrow B_{\infty}$ induces an injection in homology with any field coefficients, see [CML], and by homology stability of the non-orientable mapping class group the theorem follows. 


\section{Calculations in unstable homology}

In this section we examine homomorphism induced in unstable homology with field coefficients by our embeddings. We restrict our discussion to the orientable case though a similar analysis goes through also in the non-orientable case.

Consider any map

$$
\alpha_{*}: H_{*}\left(B_{m}, \mathbb{F}\right) \longrightarrow H_{*}\left(\Gamma_{g, b}, \mathbb{F}\right)
$$

induced by a homomorphism $\alpha: B_{m} \rightarrow \Gamma_{g, b}$ that is part of a $\mathcal{D}$-algebra map; here $b=1,2$ and $\mathbb{F}$ is any field. The main fact we will be using is that the homology of the braid group is generated by classes of degree one when taking the $\mathcal{D}$-algebra structure into account.

6.1. The rational case: Recall from $[\mathrm{CLM}]$ that for $m>1$ the $\mathbb{Q}$-homology of $B_{m}$ is of rank one in degrees 0 and 1 , and zero otherwise. Thus rationally, the braid groups have the homology of a circle. We recall

$$
H_{1}\left(\Gamma_{2,2}\right)=H_{1}\left(\Gamma_{2,1}\right)=\mathbb{Z} / 10 \mathbb{Z}, \quad \text { and } \quad H_{1}\left(\Gamma_{g, 1}\right)=0 \text { when } g \geq 3 .
$$

The first identity follows from the stability results [Bo] and [RW]; and the two computations are well-known. Thus $\alpha_{*}$ is trivial in rational homology.

We now turn to fields of finite characteristic. Recall from [CLM] that for $m>1$ the $\mathbb{F}_{p}$-homology is generated by $H_{1}\left(B_{m} ; \mathbb{F}_{p}\right)=\mathbb{F}_{p}$ and the homology operations induced from the action of $\mathcal{D}$. These operations are the product, the first Dyer-Lashof operation $Q$, and in the case of odd primes, the combination with the Bockstein operator $\beta Q$. More precisely, for $x \in H_{*}\left(B_{m} ; \mathbb{F}_{p}\right)$, the operation $Q$ is defined by the formula

$$
\begin{array}{lll}
Q(x)=\theta_{*}\left(e_{1} \otimes x \otimes x\right) & \text { for } & p=2 \\
Q(x)=\theta_{*}\left(e_{p-1} \otimes x^{p}\right) & \text { for } & p>2
\end{array}
$$

where $e_{1}$ is of degree $1, e_{p-1}$ of degree $p-1$, and $\theta_{*}$ is induced by the action $\theta=\theta_{\mathcal{B}}$ of $\mathcal{D}$ on $\mathcal{B}$ as defined in section 4 .

6.2. The case $p$ even: Quoting [CLM, p. 347], the $\mathbb{F}_{2}$-homology of the braid group can be described as

$$
H_{*}\left(B_{m} ; \mathbb{F}_{2}\right)=\mathbb{F}_{2}\left[x_{i}\right] / I
$$

where $x_{i}=Q\left(x_{i-1}\right)$ is of degree $2^{i}-1$ and $I$ is the ideal generated by all monomials $x_{i_{1}}^{k_{1}} \ldots x_{i_{t}}^{k_{t}}$ such that $\sum_{j=1}^{t} k_{j} 2^{i_{j}}>m$. In particular,

$$
x_{i}=0 \quad \text { if } \quad 2^{i}>m .
$$


6.3. The case $p$ odd: Similarly, by [CLM, p. 347], for $p$ odd the $\mathbb{F}_{p^{-} \text {-homology }}$ of the braid group is a polynomial algebra on generators $\lambda, y_{i}$, and $\beta y_{i}$ of degrees $1,2 p^{i}-1$, and $2 p^{i}-2$, modulo some ideal $J$ which includes $y_{i}$ whenever $2 p^{i}>m$. Furthermore, $y_{1}=Q(\lambda)$ and $y_{i+1}=Q\left(y_{i}\right)$.

We consider now the image of the generators $x_{i}$ and $y_{i}$ under the map $\alpha_{*}$. As an immediate consequence of Theorem 5.1, and using the best homology stability range available, we have

$$
\begin{aligned}
& \alpha_{*}\left(x_{i}\right)=0 \quad \text { for } \quad\left(3 \cdot 2^{i}-1\right) / 2 \leq g \quad \text { and } \quad p=2 \\
& \alpha_{*}\left(y_{i}\right)=0 \quad \text { for } \quad\left(6 p^{i}-1\right) / 2 \leq g \quad \text { and } \quad p>2 \text {. }
\end{aligned}
$$

We now explain in two examples how the Dyer-Lashof algebra structure can be used to deduce similar results independent of Theorem 5.1, which in some cases lead to stronger vanishing results.

6.4. Example: Consider the operadic embedding (2.5) when $m=g, b=1$ and $\alpha$ is

$$
\varphi^{+}: B_{g} \longrightarrow \Gamma_{g, 1} .
$$

The $\mathcal{D}$-algebra structure in this case is given by gluing the boundary of the surfaces $\Sigma_{g_{i}, 1}$ to the boundaries of the holes in the disk $\Sigma_{0, k+1}$. The genus of the resulting surface is simply the sum of the genera $g_{i}$.

Observe that to use the $\mathcal{D}$-algebra structure, the genus of the surface corresponding to the target group has to be large enough to be able to decompose it. The equation $\varphi_{*}^{+}(Q(z))=Q\left(\varphi_{*}^{+}(z)\right)$ gives rise to the following inductive formula. Assume $\varphi_{*}^{+}\left(x_{i+1}\right)=0$ or $\varphi_{*}^{+}\left(y_{i}\right)=0$ when the genus of the target surface is at least $d_{i}$. Then using (6.3) and (6.4) we can conclude that

$$
d_{i}=p d_{i-1} \quad \text { and thus } \quad d_{i}=p^{i} d_{0},
$$

where, by (6.2), $d_{0}=3$ when $p=2,5$, and $d_{0}=2$ when $p \neq 2,5$. Thus we have that

$$
\begin{array}{ll}
\varphi_{*}^{+}\left(x_{i}\right)=0 & \text { if } 3 \cdot 2^{i-1} \leq g \quad \text { and } p=2, \\
\varphi_{*}^{+}\left(y_{i}\right)=0 & \text { if } 3 p^{i} \leq g \quad \text { and } p=5, \\
\varphi_{*}^{+}\left(y_{i}\right)=0 & \text { if } 2 p^{i} \leq g \quad \text { and } p \neq 5 .
\end{array}
$$

This gives an improvement on (6.5) and (6.6) for primes other than 2 and 5 .

As $x_{i}=0$ for $2^{i}>m$ and $y_{i}=0$ for $2 p^{i}>m$, our computations show that the map in homology induced by $\varphi^{+}$is zero in unstable homology of positive 
degree for characteristics $p \neq 2,5$. When $p=2$ or $p=5$, we cannot always decide with the above methods whether the top dimensional $x_{i}$ and $y_{i}$ classes are mapped to zero or not.

6.5. Example: We now consider the geometric embedding (1.1) when $m=$ $2 g+2, b=2$ and $\alpha$ is

$$
\phi: B_{2 g+2} \longrightarrow \Gamma_{g, 2} \text {. }
$$

The $\mathcal{D}$-algebra structure in this case glues the surfaces $\Sigma_{g_{i}, 2}$ to two disks $\Sigma_{0, k+1}$ with $k$ holes. The resulting surface has genus the sum of the genera $g_{i}$ plus $k-1$. Thus, the inductive formula for $d_{i}$ is given by

$$
d_{i}=p d_{i-1}+p-1=p^{i}\left(d_{0}+1\right)-1 .
$$

By (6.2) we have $d_{0}=3$ and $d_{i}=4 p^{i}-1$ for $p=2,5$, and we have $d_{0}=2$ and $d_{i}=3 p^{i}-1$ for $p \neq 2,5$.

The $d_{i}$ are growing faster here then in Example 6.4. Indeed, as is easily checked, any class $x_{i}$ and $y_{i}$ that in this way can be shown to vanish under $\phi_{*}$ is already in the stable range. Thus no extra information can be gained in addition to what is known by Theorem 5.1; compare (6.5) and (6.6).

In particular, we cannot determine with our methods here whether (a) the top three $x_{i}$ classes and whether (b) the top two $y_{i}$ classes for $p=3$ resp. the top $y_{i}$ class for $p \neq 3$ vanish under $\phi_{*}$. We have $x_{i} \neq 0$ when $2^{i-1}-1 \leq g$, while $\phi_{*}\left(x_{i}\right)=0$ when $2^{i+2}-1 \leq g$ and similarly $y_{i} \neq 0$ when $p^{i-1}-1 \leq g$, while $\phi_{*}\left(y_{i}\right)=0$ when $4 p^{i}-1 \leq g$ in case $p=5$, or when $3 p^{i}-1 \leq g$ in case $p \neq 5$.

Therefore the conclusions drawn in [SeT; Corollary 4.1] (and [C; Corollary 2.7]) are too strong: It remains an open question whether for $g \geq 3$ the homomorphism $\phi: B_{2 g+2} \rightarrow \Gamma_{g, 2}$ induces the zero map in reduced $\mathbb{F}_{p}$-homology for all primes $p$.

In our explicit calculations above we have concentrated on the generators $x_{i}$ and $y_{i}$. But a similar analysis can be given to determine when the image of products is trivial.

Finally, an analogous study can be given for $\mathcal{D}$-algebra maps from braid groups to mapping class groups of non-orientable mapping class groups in the case when $p \neq 2$. Note, in that case $H_{1}\left(\mathcal{N}_{g} ; \mathbb{F}_{p}\right)=0$ for $g \geq 7$ by $[\mathrm{K}]$.

\section{References}

[A] Artin, Emil: Theorie der Zöpfe. Abh. Math. Semin. Univ. Hamburg 4 (1925) 47-72. 
[BC1] Birman, Joan S.; Chillingworth, D. R. J.: On the homeotopy group of a non-orientable surface. Proc. Cambridge Philos. Soc. 71 (1972), 437-448.

[BC2] Birman, Joan S.; Chillingworth, D. R. J.: Erratum: "On the homeotopy group of a non-orientable surface" [Proc. Cambridge Philos. Soc. 71 (1972), 437-448; MR0300288]. Math. Proc. Cambridge Philos. Soc. 136 (2004), no. 2, 441.

[BH1] Birman, Joan S.; Hilden, Hugh M.: Lifting and projecting homeomorphisms. Arch. Math. (Basel) 23 (1972), 428-434.

[BH2] Birman, Joan S.; Hilden, Hugh M.: On isotopies of homeomorphisms of Riemann surfaces. Ann. of Math. (2) 97 (1973), 424-439.

[Bo] Boldsen, Soren K.: Improved homological stability for the mapping class group with integral or twisted coefficients. arXiv:0904.3269

[Bö] Bödigheimer, Carl-Friedrich: On the topology of moduli spaces of Riemann surfaces, Part II: homology operations. Math. Gottingensis, Heft 23 (1990).

[C] Cohen, Frederick R.: Homology of mapping class groups for surfaces of low genus. The Lefschetz centennial conference, Part II (Mexico City, 1984), 21-30, Contemp. Math., 58, II, Amer. Math. Soc., Providence, RI, 1987. 
[CLM] Cohen, Frederick R.; Lada, Thomas J.; May, J. Peter: The homology of iterated loop spaces. Lecture Notes in Mathematics, Vol. 533. SpringerVerlag, Berlin-New York, 1976.

[I] Ivanov, Nikolai V.: Complexes of curves and the Teichmüller modular group. Uspekhi Mat. Nauk 42 (1987), 49-91.

[K] Korkmaz, Mustafa: First homology group of mapping class groups of nonorientable surfaces. Math. Proc. Cambridge Philos. Soc. 123 (1998), no. $3,487-499$.

[M] Miller, Edward Y.: The homology of the mapping class group. J. Differential Geom. 24 (1986), 1-14.

[PR] Paris, Luis; Rolfsen, Dale: Geometric subgroups of mapping class groups. J. Reine Angew. Math. 521 (2000), 47-83.

$[\mathrm{P}] \quad$ Powell, Jerome: Two theorems on the mapping class group of a surface. Proc. Amer. Math. Soc. 68 (1978), no. 3, 347-350.

[RW] Randal-Williams, Oscar: Resolutions of moduli spaces and homological stability. arXiv:0909.4278

[SeT] Segal, Graeme; Tillmann, Ulrike: Mapping configuration spaces to moduli spaces. Groups of diffeomorphisms, 469-477, Adv. Stud. Pure Math., 52, Math. Soc. Japan, Tokyo, 2008.

[SoT] Song, Yongjin; Tillmann, Ulrike: Braids, mapping class groups, and categorical delooping. Math. Ann. 339 (2007), no. 2, 377-393.

[S] Szepietowski, Blazej: Embedding the braid group in mapping class groups. Publ. Mat. 54 (2010), no. 2, 359-368.

[T1] Tillmann, Ulrike: Higher genus surface operad detects infinite loop spaces. Math. Ann. 317 (2000), no. 3, 613-628.

[T2] Tillmann, Ulrike: The representation of the mapping class group of a surface on its fundamental group in stable homology. Q. J. Math. 61 (2010), no. 3, 373-380.

[Wa1] Wahl, Nathalie: Infinite loop space structure(s) on the stable mapping class group. Topology 43 (2004), no. 2, 343-368.

[Wa2] Wahl, Nathalie: Homological stability for the mapping class groups of non-orientable surfaces. Invent. Math. 171 (2008), no. 2, 389-424.

[W1] Wajnryb, Bronislaw: Artin groups and geometric monodromy. Invent. Math. 138 (1999), no. 3, 563-571. 
[W2] Wajnryb, Bronislaw: Relations in the mapping class group. Problems on mapping class groups and related topics, 115-120, Proc. Sympos. Pure Math., 74, Amer. Math. Soc., Providence, RI, 2006.

Carl-Friedrich Bödigheimer

Mathematisches Institut

Universität Bonn

Endenicher Allee 60

53115 Bonn, Germany

boedigheimer@math.uni-bonn.de

Ulrike Tillmann

Mathematical Institute

Oxford University

24-29 St Giles

Oxford OX1 3LB, United Kingdom

tillmann@maths.ox.ac.uk 